\title{
Mean encounter times for cell adhesion in hydrodynamic flow: analytical progress by dimensional reduction
}

\author{
C. B. Korn and U. S. SchwarZ \\ University of Heidelberg, Bioquant 0013, Im Neuenheimer Feld 267, D-69120 Heidelberg, Germany
}

PACS 82.39.- $\mathrm{k}$ - chemical kinetics in biological systems

PACS 47.15.G- - fluid dynamics, low-Reynolds-number flows

PACS 05.10.Gg - stochastic analysis methods (FokkerPlanck, Langevin, etc.)

\begin{abstract}
For a cell moving in hydrodynamic flow above a wall, translational and rotational degrees of freedom are coupled by the Stokes equation. In addition, there is a close coupling of convection and diffusion due to the position-dependent mobility. These couplings render calculation of the mean encounter time between cell surface receptors and ligands on the substrate very difficult. Here we show for a two-dimensional model system how analytical progress can be achieved by treating motion in the vertical direction by an effective reaction term in the mean first passage time equation for the rotational degree of freedom. The strength of this reaction term can either be estimated from equilibrium considerations or used as a fit parameter. Our analytical results are confirmed by computer simulations and allow to assess the relative roles of convection and diffusion for different scaling regimes of interest.
\end{abstract}

Introduction. - Biological function is often based on the formation of a specific binding complex between receptor and ligand [1]. However, in order for binding to oc' cur, a physical transport process must exist which brings the binding partners to sufficiently close proximity [2]. In many cases of interest, this transport process is rather complex. Usually it contains several coupled degrees of freedom, like a cell surface receptor moving laterally on a membrane which fluctuates in the vertical direction [3]. The efficiency of biological transport processes often can be framed as mean first passage time (MFPT) problems, for example for the gating of ion channels [4] or the arrival of a virus at the nucleus [5]. Another example of a complex transport process of large biological relevance is the receptor-mediated adhesion of cells which are carried over a ligand-coated substrate by hydrodynamic flow [6]. Here the mean encounter time between receptors and ligands is a measure for the efficiency of cell adhesion under the conditions of hydrodynamic flow [7]. For this system, additional complications arise from the presence of multiple length scales. For the micron-sized cell, the hydrodynamic equations result in coupling of the translational and rotational degrees of freedom. Even for high shear rates, Brownian motion is relevant because receptors and ligands are nanometer-sized objects, thus even small movements for the cell result in a large effect on the molecular level.
Here we show that despite the presence of these complications, analytical progress can be achieved by dimensional reduction of appropriate degrees of freedom. The reduced description then contains effective parameters which have to be obtained from the full model.

Experimentally the binding of cells to a substrate in hydrodynamic flow is often studied in flow chambers because this setup allows for controlled flow conditions $[8,9]$. In vivo, this situation is relevant for white blood cells, which travel the body with the blood flow, but have to adhere at very specific locations, e.g. close to sites of inflammation. Similar mechanisms are used by cancer and stem cells. Moreover, malaria-infected red blood cells also undergo adhesion to the vessel walls under flow conditions. Apart from using flow chambers, one can further reduce the experimental system by employing biomimetic analogs of cells, that is receptor bearing micro-beads $[10,11]$. The efficiency with which cells or beads in flow can bind to a substrate depends crucially on the spatial distribution of receptors and ligands [12]. Previously, we proposed a model based on a Langevin equation for a spherical particle in linear shear flow above a wall which allows to numerically compute MFPTs for different ligand and receptor distributions and flow parameters both for two-dimensional (2D) and three-dimensional (3D) movements $[7,13]$. Due to the complex geometry arising from the receptor and 
ligand distributions and the complexity of the positiondependent mobility functions arising from the hydrodynamic equations, exact analytic results for the MFPT cannot be obtained in this general case.

In this letter we present analytical advances for $2 \mathrm{D}$ motion with homogeneous ligand coverage on the substrate. In our model system, motion can occur only in $\mathrm{x}$ - and $\mathrm{z}$-directions and rotation is restricted about the y-axis. Due to the assumption of homogeneous ligand coverage, motion in the $\mathrm{x}$-direction is not relevant. Thus we deal with two degrees of freedom, falling in z-direction and rotation about the y-axis. This is the simplest model system which combines rotational and translational degrees of freedom in a non-trivial manner. Because cell movement usually occurs in the regime of small Reynolds number, their coupling is determined by the Stokes equation for viscous flow. In addition, we account for Brownian motion which is ubiquitous in biological systems on the nanoscale and essential for receptor-ligand binding to occur. Here we show that this model system can be further reduced by effectively integrating out the translational degree of freedom. This results in an ordinary differential equation for the MFPT of the rotational degree of freedom which includes a non-trivial reaction term that represents the falling motion of the cell. We show that this equation can be solved analytically. From this solution we then derive various expressions that describe asymptotic limits. By comparing with computer simulations, we finally show for which parameter range our analytical results are valid. Moreover, our analytical calculations still explain the numerical results outside this parameter range when the effective reaction rate is used as a fit parameter.

Model definition. - We consider a sphere of radius $R$ moving in linear shear flow with shear rate $\dot{\gamma}$ above a planar wall. As explained above, we restrict its motion to two dimensions, that is the translational motion of the sphere is restricted to a plane perpendicular to the boundary wall, i. e. the $x z$-plane, and rotations are only allowed about the $y$ axis (see fig. 17). As depicted in fig. 1h the circumference of the sphere lying in the $x z$-plane is covered with $N_{r}$ equidistantly distributed receptor patches of height $r_{0} \ll R$ and radius $r_{p} \ll R$. The boundary wall is homogeneously covered with ligands. In order to drive it onto the substrate, in vertical direction the sphere is subject to a constant force $-F_{z}$. In experiments, this force arises from gravity because cells or micro-beads are usually slightly denser than the surrounding medium. A receptor-ligand encounter occurs with certainty whenever a receptor patch has some overlap with the boundary wall. Because we also consider Brownian motion, the receptorligand encounter is stochastic. Our goal is to calculate the corresponding MFPT. For the calculation of first passage times the motion in $x$ direction can be neglected as the system is translationally invariant in this direction due to homogeneous ligand coverage. Moreover, the regular distribution of receptor patches generates a $\theta_{s}:=2 \pi / N_{r}$ symmetry for the $\theta$ coordinate (i.e., the angle about the $y$-axis). Therefore, we deal with the situation illustrated in fig. 1b of a diffusive particle moving in the $\theta_{s}$-periodic $(z \theta)$ plane (i.e., a cylindrical surface with $z>R$ ) with absorbing boundaries $\theta(z)$ (solid lines in fig. 1) representing the boundaries of the encounter areas.

If $T\left(z^{\prime}, \theta^{\prime}\right)$ is the MFPT to reach a point on the absorbing boundary when started at some point $\left(z^{\prime}, \theta^{\prime}\right)$ then for practical purposes only $\left\langle T\left(z^{\prime}, \theta^{\prime}\right)\right\rangle_{\theta^{\prime}}$, i. e., the MFPT averaged over all initial orientations, is a relevant quantity as it is experimentally very difficulty to prepare a certain initial orientation. Concerning the initial height we previously showed [13] that for $z^{\prime}>z_{m}>R+r_{0}$ the following relation holds true

$$
\left\langle T\left(z^{\prime}, \theta^{\prime}\right)\right\rangle_{\theta^{\prime}}=T\left(z_{m} \mid z^{\prime}\right)+\left\langle T\left(z_{m}, \theta_{m}\right)\right\rangle_{\theta_{m}},
$$

where $T\left(z_{m} \mid z^{\prime}\right)$ is the mean time to fall from the initial height $z^{\prime}$ to the intermediate height $z_{m}$ and $\theta_{m}$ is the sphere's orientation at height $z_{m}$. Eq. (1) states that if the angle averaged MFPT is known for some height $z_{m}$ the MFPT can be calculated for any other height $z>z_{m}$ without further considering the orientational degree of freedom. Moreover $T\left(z_{m} \mid z^{\prime}\right)$ can be calculated exactly [13]. Thus for the following we choose as the initial height of the disk always $z^{\prime}=R+r_{0}$ and write $T\left(\theta^{\prime}\right):=T\left(z^{\prime}=R+r_{0}, \theta^{\prime}\right)$.

Model reduction to one dimension. - In order to calculate $\left\langle T\left(\theta^{\prime}\right)\right\rangle_{\theta^{\prime}}$ one must in principle consider the motion in the full $(z \theta)$-plane. Here, we show that under certain conditions a good approximation formula for this MFPT can be derived by considering only the motion of a particle in a periodic one-dimensional $\theta$-space. For that we first approximate the area in the $(z \theta)$-plane in which encounter occurs by a rectangle of width $2 \theta_{0}$ (see fig. 10). As an appropriate $\theta_{0}$ we choose the mean width of the encounter interval the particle sees while being at height $z<R+r_{0}$, that is

$$
\theta_{0}=\frac{1}{1-e^{-F_{z} r_{0} / k_{B} T_{a}}} \int_{R}^{R+r_{0}} \mathrm{~d} z \theta_{0}(z) \Psi_{s}(z),
$$

where

$$
\Psi_{s}(z)=\frac{F_{z}}{k_{B} T_{a}} e^{-F_{z}(z-R) / k_{B} T_{a}}
$$

is the stationary probability distribution for the height of the sphere $z$ with Boltzmann's constant $k_{B}$ and ambient temperature $T_{a} \cdot \theta_{0}(z)$ is the boundary of the encounter area and depends on receptor height and radius through $\theta_{0}(z)=\arccos \left(z /\left(R+r_{0}\right)\right)+r_{p}$ [13]. Thus $\theta_{0}$ increases with $r_{p}$.

Changes in $\theta$ are due to rotational diffusion with diffusion coefficient $D_{\theta}$ and a drift $A_{\theta} \propto \dot{\gamma}$ arising from the linear shear flow. This suggests to take the corresponding Fokker-Planck equation as a starting point for a reduced model. In order to account for the effect of the motion in z-direction, we argue that for $\theta \in\left[0,2 \theta_{0}\right]$ an encounter 


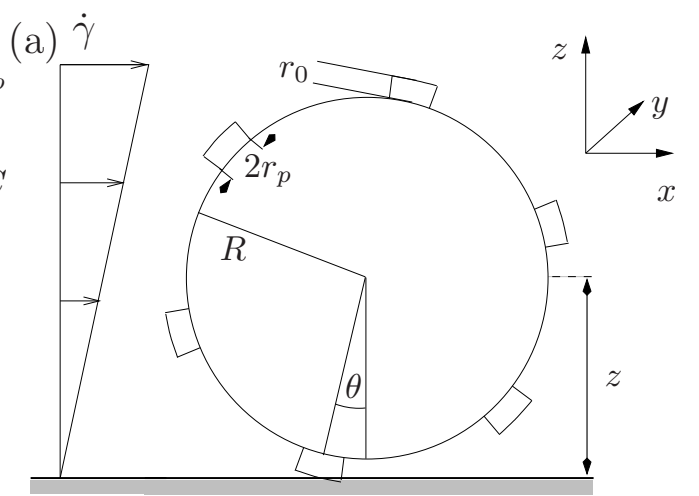

(b)

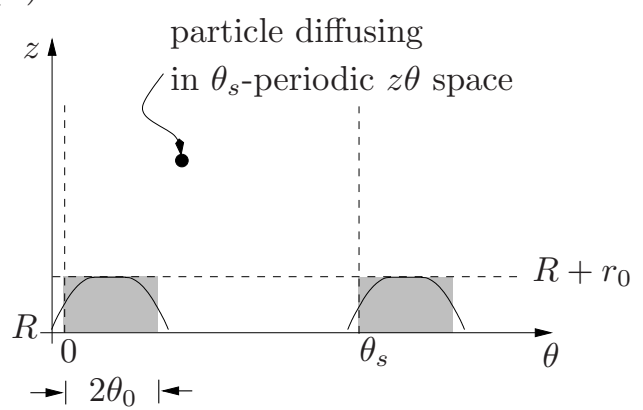

Fig. 1: (a) A sphere (radius $R$ ) in linear shear flow (shear rate $\dot{\gamma})$ covered with $N_{r}=6$ receptor patches with height $r_{0}$ and radius $r_{p}$ is shown. The sphere translates in the $x z$-plane. The orientation about the symmetry axis is given by the angle $\theta$. (b) The encounter problem can be mapped to that of a particle moving in $z \theta$ space, where the motion in $\theta$-direction is $\theta_{s}:=$ $2 \pi / N_{r}$ periodic as the receptors are equidistantly distributed. The solid curves shown enclose the areas in which receptorligand encounter occurs. For the calculation we approximate these areas by a rectangular area (grey shaded) of height $r_{0}$ and width $2 \theta_{0}$.

occurs only with a finite probability. Thus, for the probability $p(\theta, t)$ to have the orientation $\theta$ at time $t$ we setup the following reaction-advection-diffusion equation for the interval $\left[0, \theta_{s}[\right.$

$$
\partial_{t} p(\theta, t)=D_{\theta} \partial_{\theta}^{2} p(\theta, t)-A_{\theta} \partial_{\theta} p(\theta, t)-\bar{k}(\theta) p(\theta, t) .
$$

The reaction term is defined by $\bar{k}(\theta)=\bar{k}$ for $\theta \in\left[0,2 \theta_{0}\right]$ and $\bar{k}(\theta)=0$ otherwise. It accounts for the finite probability that encounter occurs while the particle is passing the interval $\left[0,2 \theta_{0}\right]$. This approximation makes sense if $\theta_{0}$ is small and if in addition $\theta_{0} / \theta_{s} \ll 1$. In our context, this assumption is justified because we have the separation of length scales $r_{p}, r_{0} \ll R$. Then the time for passing the interval $\left[0,2 \theta_{0}\right]$ is short compared to the time between successive receptor-ligand encounter and the correlation between the heights $z$ for successive encounters are small, thus justifying our mean field treatment for the translational degree of freedom. The assumption $\theta_{0} / \theta_{s} \ll 1$ also suggests to take the limit $\theta_{0} \rightarrow 0$ while keeping $2 \theta_{0} \bar{k}=: k$ constant. Then the term $-\bar{k}(\theta) p(\theta, t)$ in eq. (4) becomes $-k \delta(\theta) p(\theta, t)$. eq. (4) is a differential equation for the rotational motion in which the effect of vertical motion has been absorbed into the new model parameter $k$.

Boundary conditions. - Because of the $\theta_{s^{-}}$ periodicity the probability $p(\theta, t)$ must fulfil periodic boundary conditions, i. e. $p\left(\theta+\theta_{s}, t\right)=p(\theta, t)$. Furthermore, integrating eq. (4) over a full period $\theta_{s}$ we obtain for the total loss of probability

$$
\frac{d}{d t} \int_{\tilde{\theta}}^{\tilde{\theta}+\theta_{s}} d \theta p(\theta, t)=-k p(0, t),
$$

i. e. $k$ denotes the rate of absorption at the boundary. In the limit $k \rightarrow \infty$ we have purely absorbing boundaries with $p(0, t)=p\left(\theta_{s}, t\right)=0$. On the other hand integrating eq. (4) over the open interval $] 0, \theta_{s}[$ we obtain

$$
\frac{d}{d t} \lim _{\epsilon \rightarrow 0} \int_{0+\epsilon}^{\theta_{s}-\epsilon} d \theta p(\theta, t)=J(0+, t)-J\left(\theta_{s}-, t\right)
$$

with the probability current $J(\theta, t):=-\left(D_{\theta} \partial_{\theta}-\right.$ $\left.A_{\theta}\right) p(\theta, t)$. Combining eq. (5) and eq. (6) we see that the reactive delta-function at $\theta=0$ is equivalent to so-called radiation boundaries [14]

$$
J\left(\theta_{s}, t\right)-J(0, t)=k p(0, t) .
$$

In the limit of zero encounter probability, i. e. $k=0$, the probability flux leaving the interval on the right boundary is equal to that entering the interval at the left boundary and the total probability is conserved.

Mean first passage time. - Let $G\left(\theta^{\prime}, t\right)$ denote the survival probability at time $t$, i.e. the probability that no encounter has occurred until time $t$ under the condition that the initial orientation of the particle at time $t^{\prime}=0$ was $\theta^{\prime}$. With $p\left(\theta, t \mid \theta^{\prime}, 0\right)$ being the conditional probability for the particle of having the orientation $\theta$ at time $t$ when the initial orientation of the particle at $t^{\prime}=0$ was $\theta^{\prime}$, the survival probability can be written as $G\left(\theta^{\prime}, t\right)=\int_{0}^{\theta_{s}} \mathrm{~d} \theta p\left(\theta, t \mid \theta^{\prime}, 0\right) . G\left(\theta^{\prime}, t\right)$ obeys the adjoint equation $[15]$

$$
\partial_{t} G\left(\theta^{\prime}, t\right)=\left(D_{\theta} \partial_{\theta^{\prime}}^{2}+A_{\theta} \partial_{\theta^{\prime}}-k \delta\left(\theta^{\prime}\right)\right) G\left(\theta^{\prime}, t\right) .
$$

The mean first passage time $T\left(\theta^{\prime}\right)$ follows from the survival probability as $T\left(\theta^{\prime}\right)=\int_{0}^{\infty} \mathrm{d} t G\left(\theta^{\prime}, t\right)$. Thus, an equation for the MFPT is obtained by integrating eq. (8) over all times. With $\lim _{t \rightarrow \infty} G\left(\theta^{\prime}, t\right)=0$ and $G\left(\theta^{\prime}, 0\right)=1$ the MFPT is the solution of [15]

$$
\left(D_{\theta} \partial_{\theta^{\prime}}^{2}+A_{\theta} \partial_{\theta^{\prime}}-k \delta\left(\theta^{\prime}\right)\right) T\left(\theta^{\prime}\right)=-1 .
$$

The general solution of eq. (9) is given by

$$
T\left(\theta^{\prime}\right)=-\frac{\theta^{\prime}}{A_{\theta}}+\frac{D_{\theta}}{A_{\theta}^{2}}\left(1-a_{1} e^{-\frac{A_{\theta}}{D_{\theta}} \theta^{\prime}}\right)+a_{2},
$$


with two integration constants $a_{1}, a_{2}$ which have to be determined in order to match the boundary conditions, i.e. periodicity $T(0)=T\left(\theta_{s}\right)$. The condition corresponding to eq. (7) is $\left.\partial_{\theta^{\prime}} T\right|_{\theta^{\prime}=0}-\left.\partial_{\theta^{\prime}} T\right|_{\theta^{\prime}=\theta_{s}}=\left(k / D_{\theta}\right) T(0)$, which follows from integrating eq. (9) from $-\epsilon$ to $\epsilon$ with some $\epsilon>0$ and then taking the limit $\epsilon \rightarrow 0$. This gives

$$
T\left(\theta^{\prime}\right)=\frac{\theta_{s}}{A_{\theta}} \frac{1-e^{-\frac{A_{\theta}}{D_{\theta}} \theta^{\prime}}}{1-e^{-\frac{A_{\theta}}{D_{\theta}} \theta_{s}}}-\frac{\theta^{\prime}}{A_{\theta}}+\frac{\theta_{s}}{k} .
$$

This equation has to be corrected by the physical expectation that $T\left(\theta^{\prime}\right)=0$ for $\theta^{\prime} \in\left[0,2 \theta_{0}\right]$ as we used for the initial height always $z^{\prime}=R+r_{0}$. Therefore, for these orientations encounter occurs instantaneously. This problem is fixed by replacing $\theta_{s}$ by $\Delta \theta:=\theta_{s}-2 \theta_{0}$ in eq. (11). Then averaging over all initial orientations including some for which $T$ is zero we arrive at our central result

$$
\begin{aligned}
\left\langle T\left(\theta^{\prime}\right)\right\rangle_{\theta^{\prime}} & =\frac{1}{\theta_{s}} \int_{0}^{\Delta \theta} \mathrm{d} \theta^{\prime} T\left(\theta^{\prime}\right) \\
& =\frac{A_{\theta} \Delta \theta^{2} \operatorname{coth}\left(\frac{A_{\theta} \Delta \theta}{2 D_{\theta}}\right)-2 D_{\theta} \Delta_{\theta}}{2 A_{\theta}^{2} \theta_{s}}+\frac{\Delta \theta^{2}}{k \theta_{s}} .
\end{aligned}
$$

As one would expect $\left\langle T\left(\theta^{\prime}\right)\right\rangle_{\theta^{\prime}}$ becomes the smaller the larger the encounter probability, i.e. the larger the reaction rate $k$.

Parameter estimates. - In order to apply eq. (12) to the situation of a particle moving in the $(z \theta)$-plane we still have to provide expressions for the reaction rate $k$ as well as for the diffusion constant $D_{\theta}$ and the drift $A_{\theta}$. Regarding the reaction rate, we have to consider the full system again. We first note that in the stationary state the probability for the sphere to be at a height between $z$ and $z+\mathrm{d} z$ is given by $\Psi_{s}(z) \mathrm{d} z$ following from eq. (3). Thus, we get for the probability for encounter while the sphere is oriented such that $\theta \in\left[0,2 \theta_{0}\right]$ (valid in the limit $\theta_{0} \ll 1$ ) $p_{z}:=1-\exp \left(-F_{z} r_{0} / k_{B} T_{a}\right)$. On the other hand using the originally introduced rate $\bar{k}$ the mean probability for encounter is $p_{\bar{k}}:=1-\exp (-\bar{k} \tau)$ where $\tau$ is the mean time it takes the particle to pass the interval of length $2 \theta_{0}$. For purely diffusive motion $A_{\theta}=0$ we estimate $\tau=\theta_{0}^{2} / 2 D_{\theta}$ which is the mean first passage time to reach the boundary of $\left[0,2 \theta_{0}\right]$ when initially started at $\theta(t=0)=\theta_{0}$. In the limit of very large drift motion becomes purely deterministic and we expect $\tau=2 \theta_{0} / A_{\theta}$. These two limiting cases may be combined to provide $1 / \tau=2 D_{\theta} / \theta_{0}^{2}+A_{\theta} / 2 \theta_{0}$. Under the assumption that the position between two successive approaches of the interval $\left[0,2 \theta_{0}\right]$ is independent one can get an estimate for $k$ from the condition $p_{\bar{k}}=p_{z}$

$$
k=\frac{F_{z} r_{0}}{k_{B} T_{a}} \frac{2 \theta_{0}}{\tau}=\frac{F_{z} r_{0}}{k_{B} T_{a}}\left(\frac{4 D_{\theta}}{\theta_{0}}+A_{\theta}\right),
$$

where we used $k=2 \theta_{0} \bar{k}$. From eq. (13) one sees that encounter becomes the more probable the larger the vertical drift force $F_{z}$ and the faster rotation given by $D_{\theta}$ and $A_{\theta}$.
For a sphere in linear shear flow above a wall the coefficients $D_{\theta}$ and $A_{\theta}$ depend on the height $z$ as

$$
D_{\theta}(z)=\frac{k_{B} T_{a}}{8 \pi \eta R^{3}} \tilde{\beta}^{r r}(R / z), \quad A_{\theta}(z)=\frac{\dot{\gamma}}{2}\left(1-\tilde{\beta}^{d r}(R / z)\right),
$$

with $\eta$ denoting the viscosity of the fluid. $\tilde{\beta}^{r r}(1 / z)$ and $\tilde{\beta}^{d r}(R / z)$ are dimensionless functions including the $z$ dependence. A numerical scheme that allows to accurately calculate these functions at arbitrary heights can be found in $[16,17]$. In order to compare the results of the model presented here with the numerical solutions of the MFPT problem we use mean-field values in the following way

$$
D_{\theta}:=\int_{R}^{\infty} \mathrm{d} z \Psi_{s}(z) D_{\theta}(z), \quad A_{\theta}:=\int_{R}^{\infty} \mathrm{d} z \Psi_{s}(z) A_{\theta}(z) .
$$

That is we take for $D_{\theta}$ and $A_{\theta}$ averages with respect to the stationary probability function $\Psi_{s}(z)$ from eq. (3) of the $z$-dependent quantities $D_{\theta}(z), A_{\theta}(z)$ defined in eq. (14).

Comparison to simulation results. - In fig. 2 we compare our results for the angle averaged $\operatorname{MFPT}\langle T\rangle_{\theta^{\prime}}$ as obtained from the analytical calculation (solid lines) and from computer simulations (symbols). For this purpose we rescale time in units of the diffusive time scale $6 \pi \eta R^{3} / k_{B} T_{a}$. In fig. 2a $\langle T\rangle_{\theta^{\prime}}$ is shown as a function of the Péclet number $P e:=6 \pi \eta R^{3} \dot{\gamma} / k_{B} T_{a}$ (other parameters are defined in the figure caption). The Péclet number is a dimensionless measure for the relative importance of deterministic and diffusive motion and $A_{\theta} \propto P e$. In the case of zero rotational drift, $A_{\theta}=0, P e=0$ and motion is purely diffusive. In addition to the simulation results (symbols) fig. 2 a also shows our main result (lines) eq. (12) where we used eqs. (13) and (15) for the rate $k$ and the constants $A_{\theta}, D_{\theta}$, respectively. The agreement between the mean-field and simulation results is surprisingly good for the parameter values chosen. In particular, even the small shoulder for $N_{r}=1$ seems to be reproduced by the analytical result. In fig. $2 \mathrm{~b}\langle T\rangle_{\theta^{\prime}}$ is shown as a function of the number of receptor patches $N_{r}$ in the diffusive limit $A_{\theta}=0$ and for two different values of the patch height $r_{0}$. Whereas the theoretical approximation with the rate given by eq. (13) works quite well for $r_{0}=10^{-3} R$, for a tenfold larger $r_{0}$ we notice clear deviations. The larger $r_{0}$ the larger $\theta_{0}$ and the assumption of the dwelling time within $\left[0,2 \theta_{0}\right]$ being small is less valid. Nevertheless, eq. (12) describes the functional dependence of $\left\langle T\left(N_{r}\right)\right\rangle_{\theta^{\prime}}$ in a qualitative way. In fact quantitative agreement can be achieved by using the reaction rate $k$ as a fit parameter (not shown).

Asymptotic limits. - The results shown in fig. 2 were obtained for a fixed vertical drift with strength given by $P e_{z}=50$. Here, $P e_{z}:=F_{z} R / k_{B} T_{a}$ is the Péclet number for the $z$-direction. Fig. 3 a displays the comparison between the numerical result of $\langle T\rangle_{\theta^{\prime}}$ and the approximation eq. (12) as a function of $P e_{z}$ in the diffusive limit $A_{\theta} \approx 0$. 

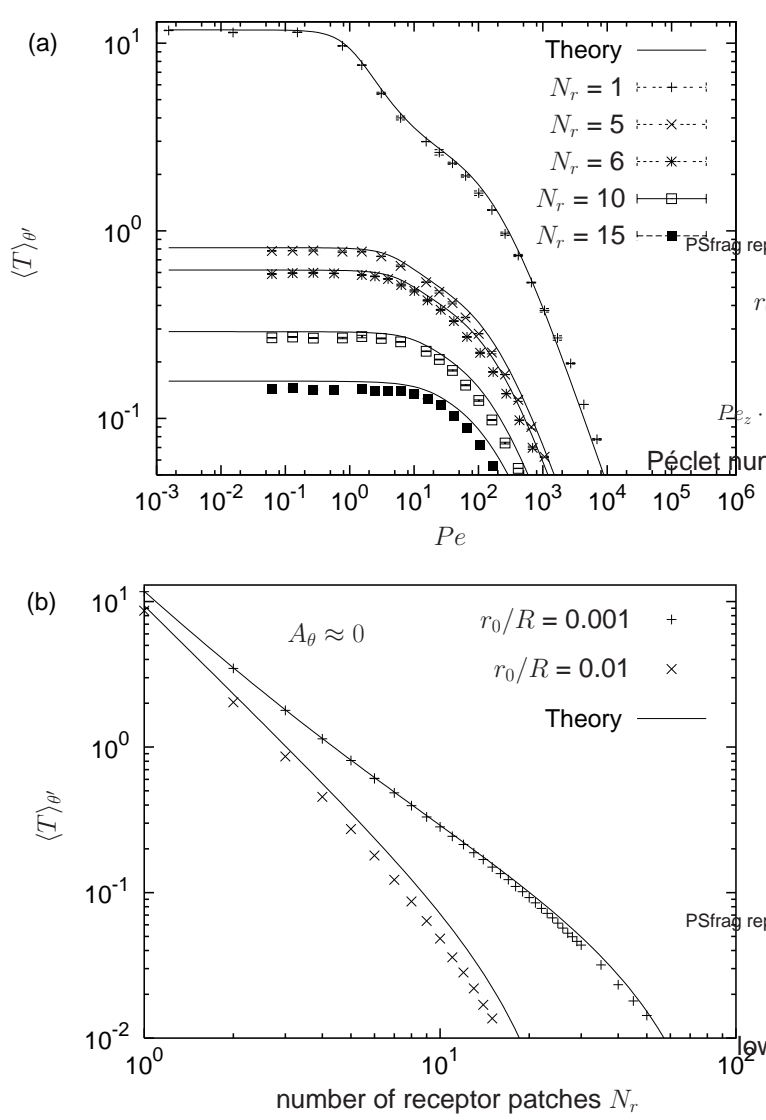

Fig. 2: Comparison between numerical results for the MFPT $\langle T\rangle_{\theta^{\prime}}$ averaged over all initial orientations and the mean-field approximation according to eqs. (12/13/15). The numerical results where obtained as described in ref. [13] for an initial height $z=2 R$. In order to display the MFPT with initial height $z=R+r_{0}$ the falling time $T\left(R+r_{0} \mid 2 R\right)$ was subtracted according to eq. (11). (a) $\langle T\rangle_{\theta^{\prime}}$ as a function of the dimensionless Péclet number $P e$ for different numbers of receptor patches $N_{r}$. (b) $\langle T\rangle_{\theta^{\prime}}$ as a function of $N_{r}$ for two different values of the patch height $r_{0}$. (Other parameters: patch radius $r_{p}=0.001 R$, vertical drift $F_{z}=P e_{z} R / k_{B} T_{a}$ with $P e_{z}=50$.)

For small $P e_{z}$ the numerical results for the MFPT shows a $1 / P e_{z}$ scaling behaviour as indicated by the dashed line in fig. 3 k. For larger $P e_{z},\langle T\rangle_{\theta^{\prime}}$ plateaus. At even larger $P e_{z}$ the MFPT increases with increasing $P e_{z}$ (not shown) as $D_{\theta}(z) \rightarrow 0$ for $z \rightarrow R$ [17]. One sees that the approximation eq. (12) underestimates the numerical result for small values of $P e_{z}$ and overestimates it for large $P e_{z}$. For intermediate values of $P e_{z}$ of the order of 10-100 we find good agreement between the two results as demonstrated before in fig. 2 For large $P e_{z} \approx 10^{3}$ the numerical result provides $\langle T\rangle_{\theta^{\prime}} \approx 0.1$. About the same value is given by the first term in eq. (12). This means that in the diffusive limit and for large $P e_{z}$ an encounter occurs almost with probability one for $\theta \in\left[0,2 \theta_{0}\right]$. This is plausible as then the duration time for $\theta \in\left[0,2 \theta_{0}\right]$ is long enough such that the particle will most probably encounter a height
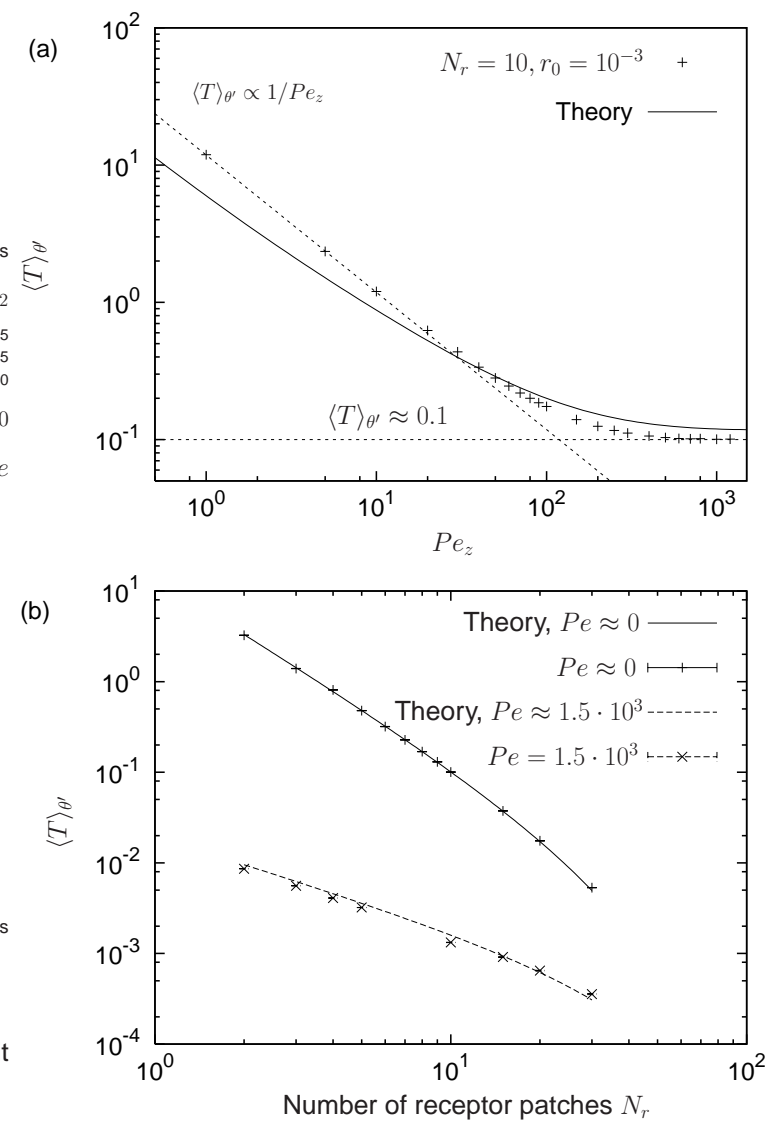

Fig. 3: (a) The numerically obtained MFPT $\langle T\rangle_{\theta^{\prime}}$ as a function of the strength of the vertical drift $P e_{z}(+)$. The dashed lines show the asymptotic behaviour $\langle T\rangle_{\theta^{\prime}} \propto 1 / P e_{z}$ for small $P e_{z}$ and the value of $\langle T\rangle_{\theta^{\prime}}$ around $P e_{z}=10^{3}$. The solid line shows the mean-field approximation according to eqs. (12)13[15). $\left(N_{r}=10, r_{0}=r_{p}=10^{-3} R\right)$. (b) The different scaling behaviour of $\langle T\rangle_{\theta^{\prime}}$ in regard to the number of receptor patches $N_{r}$ for zero and large rotational drift $A_{\theta}$ at $P e_{z}=10^{3}$ is shown. The theoretical result from eq. (12) matches well the numerical results (symbols) when for $k$ infinity and $k=2 A_{\theta}$ is chosen for $P e=0$ and $P e \approx 1500$, respectively $\left(r_{0}=r_{p}=10^{-3} R\right)$.

$z<R+r_{0}$ while the receptor patch points downwards. Therefore, the rate $k$ for $P e_{z} \approx 10^{3}$ is rather infinity than the value given by the estimate eq. (13). At small values of $P e_{z}$ we expect combining eq. (12) and eq. (13) the second term in eq. (12) to be dominant and $\langle T\rangle_{\theta^{\prime}} \propto 1 /\left(D_{\theta} P e_{z}\right)$. As the diffusion coefficient $D_{\theta}(z)$ is a monotonically increasing with increasing distance $z$ from the wall also the term $D_{\theta}$ defined in eq. (15) becomes larger with decreasing $P e_{z}$. This is the reason why the estimate eq. (12) for $\langle T\rangle_{\theta^{\prime}}$ does not provide the right scaling behaviour. On the other hand using $\left.D_{\theta}\right|_{P e_{z}=50}$ also at larger $P e_{z}$ values a much better agreement (not shown) between the theoretical estimate and the numerical result of the MFPT is obtained. That implies that the faster rotations far away from the wall, which are included in the definition eq. (15), are not relevant for the encounter process which happens only very close to the wall. 
In fig. 3b we demonstrate that eq. (12) matches well to the numerically obtained MFPT for $A_{\theta}=0$ and large $P e_{z}$ when $k$ is set to infinity as discussed above. There, for $P e_{z}=10^{3}$, the numerical result for $\langle T\rangle_{\theta^{\prime}}(+)$ and the theoretical result eq. (12) (full line) are shown. For the dependence on the number of receptor patches $N_{r}$ we find in this limit (and for $N_{r}$ small)

$$
\langle T\rangle_{\theta^{\prime}} \approx \frac{\Delta \theta^{3}}{12 \theta_{s} D_{\theta}} \approx \frac{4 \pi^{2}}{12 D_{\theta} N_{r}^{2}},
$$

i.e. the MFPT approximately scales as $1 / N_{r}^{2}$. The situation is different in the deterministic limit, i.e. for large $A_{\theta}$. Then, eq. (12) is approximately

$$
\langle T\rangle_{\theta^{\prime}} \approx \frac{\Delta \theta^{2}}{\theta_{s}}\left(\frac{1}{2 A_{\theta}}+\frac{1}{k}\right) \approx \frac{\pi}{N_{r}}\left(\frac{1}{2 A_{\theta}}+\frac{1}{k}\right),
$$

i.e. the MFPT scales as $1 / N_{r}$ at small numbers of receptor patches $N_{r}$. In fig. 3b we also show a comparison between eq. (17) (dashed line) and the numerically obtained MFPT $(\times)$. Here we find that good agreement is obtained when we choose $k \approx 2 A_{\theta}$, which is of the same order of magnitude as the estimate given by eq. (13). Thus, in contrast to the diffusive limit, the second and the first term in eq. (12) are of the same order.

Summary and outlook. - In this letter we have derived an approximate expression eq. (12) for the angle averaged MFPT for receptor-ligand encounter between a sphere equidistantly covered with receptor patches and a wall homogeneously covered with ligands in a 2D geometry. The main idea of our analysis was to integrate out the motion in $z$-direction by absorbing falling in a reaction term for the rotational degree of freedom. The coefficients for the diffusion and drift terms of this equation were estimated from mean field arguments in eq. (15). Our derivation was based on the central assumption that the heights of the sphere at two successive times of a receptor patch pointing downwards is uncorrelated. This is not true in general, and accordingly the result derived for the reaction rate $k$ in eq. (13) is valid only for a small range of parameter values. However, the rate $k$ can also be viewed as a fit parameter. In that case eq. (12) matches the results obtained in computer simulations of the full problem over a large range of parameters.

In the future, our analysis could be extended in different ways. Here we have only considered a homogeneous ligand density. For non-homogeneous ligand coverage $\rho_{l}<1$ not every receptor-wall encounter is a productive receptorligand encounter. This might again be expressed by an appropriate choice of the rate $k$ with $k \propto \rho_{l}$. Even for homogeneous ligand density, not every receptor-ligand encounter has to lead to functional adhesion under flow. Depending on the receptor-ligand system under consideration, additional steps might be required to achieve a stable bond. Conceptually, one could regard the first encounter as formation of an encounter complex [2]. The next step would then be the transition into a final complex. In a such a two-state system, also unbinding becomes important, as it characterizes the stability of the final complex. Again this process might be expressed by an appropriate choice of the rate $k$ as used here. If complete unbinding occurs, there is also the possibility that the cell forms a new bond downstream of the old one. At sufficient densities of receptors and ligands, this mechanism eventually leads to the physiologically very relevant process of rolling adhesion. Recently we have extended the computer simulations used above to measure MFPTs to also simulate the process of rolling adhesion [18]. Future work has to show how these simulations can now be made more efficient using the dimensional reduction introduced here. In general, analytical progress by dimensional reduction and introduction of appropriate reaction terms might be a very promising strategy also for other biological systems which involve a complex interplay between different transport modes.

$$
* * *
$$

This work was supported by the Center for Modelling and Simulation in the Biosciences (BIOMS) and the Cluster of Excellence CellNetworks at Heidelberg.

\section{REFERENCES}

[1] Alberts B., Johnson A., Lewis J., Raff M., Roberts K. and Walter P., Molecular Biology of the Cell 5th Edition (Garland Science) 2008.

[2] Berg H. C. and Purcell E. M., Biophys. J., 20 (1977) 193.

[3] Reister E. and Seifert U., Europhys. Lett., 71 (2005) 859.

[4] Goychuk I. and Hanggi P., Proc. Natl. Acad. Sci. USA, 99 (2002) 3552.

[5] Holcman D., J. Stat. Phys., 127 (2007) 471.

[6] Lawrence M. B. and Springer T. A., Cell, 65 (1991) 859.

[7] Korn C. and Schwarz U. S., Phys. Rev. Lett., 97 (2006) 138103.

[8] Alon R., Chen S., Puri K. D., Finger E. B. and Springer T. A., J. Cell Biol., 138 (1997) 1169.

[9] Chen S. and Springer T. A., Proc. Natl. Acad. Sci. USA, 98 (2001) 950.

[10] Pierres A., Feracci H., Delmas V., Benoliel A.-M., Thiery J.-P. and Bongrand P., Proc. Natl. Acad. Sci. USA, 95 (1998) 9256.

[11] Greenberg A. W., Brunk D. K. and Hammer D. A., Biophy. J., 79 (2000) 2391.

[12] Yago T., Zarnitsyna V. I., Klopocki A. G., McEver R. P. and Zhu C., Biophys. J., 92 (2007) 330.

[13] Korn C. B. and Schwarz U. S., J. Chem. Phys., 126 (2007) 095103.

[14] Szabo A., Schulten K. and Schulten Z., J. Chem. Phys., 72 (1980) 4350.

[15] Honerkamp J., Stochastic Dynamical Systems (VCH Publishers, Inc.) 1994. 
[16] Perkins G. S. and Jones R. B., Physica A, 189 (1992) 447.

[17] Cichocki B. and Jones R. B., Physica A, 258 (1998) 273.

[18] Korn C. B. and Schwarz U. S., Phys. Rev. E, 77 (2008) 041904. 